\title{
Spectral Efficiency Optimization in Flexi-Grid Long-Haul Optical Systems
}

\author{
Tommaso Foggi, Giulio Colavolpe, Senior Member, IEEE, Alberto Bononi, Senior Member, IEEE, \\ and Paolo Serena, Member, IEEE
}

\begin{abstract}
Flexible grid optical networks allow a better exploitation of fiber capacity, by enabling a denser frequency allocation. A tighter channel spacing, however, requires narrower filters, which increase linear intersymbol interference (ISI), and may dramatically reduce system reach. Commercial coherent receivers are based on symbol by symbol detectors, which are quite sensitive to ISI. In this context, Nyquist spacing is considered as the ultimate limit to wavelength-division multiplexing (WDM) packing. In this paper, we show that by employing a limited-complexity trellis processing at the receiver, either the reach of Nyquist WDM flexi-grid networks can be significantly extended, or a denser-than-Nyquist channel packing [i.e., a higher spectral efficiency (SE)] is possible at equal reach. By adopting well-known information-theoretic techniques, we design a limited-complexity trellis processing and quantify its SE gain in flexi-grid architectures where wavelength selective switches over a frequency grid of $12.5 \mathrm{GHz}$ are employed.
\end{abstract}

Index Terms-Coherent detection, flexi-grid, nonlinear propagation, nyquist-WDM, optical communications, polarization-multiplexed quaternary phase-shift keying, ROADM, time-frequency packing.

\section{INTRODUCTION}

$\mathbf{S}$ PURRED by the relentless increase of data traffic, coherent optical systems were revived in the last decade, and many different paths were undertaken in order to better exploit the huge capacity of the fiber channel, from polarizationmultiplexing (PM) and high-order modulations, to dense wavelength division multiplexing (WDM) solutions like NyquistWDM or orthogonal frequency division multiplexing [1], [2]. Although to cope with the foreseen capacity crunch of the existing fiber infrastructure [3] the long-term solution will likely be the use of multimode fibers and multi-input-multi-output processing - a solution that requires replacing the existing fiber infrastructure-yet a less disruptive step towards increasing the spectral efficiency (SE) has been the introduction of flexigrid WDM networks [4] where throughput increase is achieved

Manuscript received October 23, 2014; revised March 2, 2015; accepted March 2, 2015. Date of publication March 12, 2015; date of current version May 22, 2015. The paper is submitted in part at the IEEE International Conference on Communications (ICC 2015), London, U.K., June 2015. This work was supported in part by CNIT and by the Italian Ministero dell'Istruzione, dell'Università e della Ricerca (MIUR) under the FIRB project Coherent Terabit Optical Networks (COTONE).

T. Foggi is with CNIT Research Unit, I-43124 Parma, Italy (e-mail: tommaso.foggi@nemo.unipr.it).

G. Colavolpe, A. Bononi, and P. Serena are with the Dipartimento di Ingegneria dell'Informazione, Università degli Studi di Parma, I-43124 Parma, Italy (e-mail: giulio@unipr.it; alberto.bononi@unipr.it; paolo.serena@unipr.it).

Color versions of one or more of the figures in this paper are available online at http://ieeexplore.ieee.org.

Digital Object Identifier 10.1109/JLT.2015.2409981 through the reduction of channel spacing. However, the deployment of new generation flexible wavelength selective switches (WSS) [5] in reconfigurable optical add-and-drop multiplexers (ROADM), compatible with the aforementioned standard, entails a careful redesign of optical systems, and specifically transmission and reception techniques. In fact, the presence of WSS with $12.5 \mathrm{GHz}$ granularity prevents the SE increase through the simple adoption of Nyquist-shaped signaling, as the effect of crossing ROADMs, and therefore cascaded WSS filtering, is detrimental even after a few nodes [6].

We employ a maximum-a-posteriori (MAP) symbol detector [7] with a minimum number of states in order to mitigate the effects of WSS in-line filtering, and compare it to the conventional symbol-by-symbol detector. The comparison is carried out for a polarization-multiplexed quaternary phase shift keying (QPSK) modulation, both in terms of pre forward-errorcorrection (FEC) bit error rate (BER) (or equivalently Q-factor) and in terms of achievable information rate (AIR), and thus achievable SE, by resorting to the simulation-based technique detailed in [8]. While AIR represents a theoretical value that may be achieved by some optimal FEC, we next extend the analysis by equipping our receivers with existing low-density parity check codes (LDPC) designed for satellite links [9], and check their SE against that obtained from AIR. The SE of such practical LDPCs turns out to be close to the value of the achievable SE, thus providing evidence of the practical meaning of the achievable SE as an upper bound on SE of all FECs working with such a receiver. We prove, for instance, that even two-state MAP symbol detectors ${ }^{1}$ allow to more than double the maximum reach when using 25\% overhead FECs and a Nyquist channel spacing with tight optical filtering. Next, with the same MAP receiver, we show that it is possible to further increase the system SE by going beyond the Nyquist limit [10], i.e., adopting the time-frequency packing (TFP) technique, hence transmitting at a higher symbol rate at the same spacing. Results show that, by taking the standard symbol-by-symbol threshold detector at the lowest symbol rate as a reference, a gain up to almost $50 \%$ in SE (i.e., 50\% more throughput) is possible. TFP technique can also be applied to higher-order modulations, but at the expense of a great complexity increase and a corresponding small SE gain, as explained in [11]. For this reason, we believe that this technique deserves a significant interest in optical systems when applied jointly with the QPSK modulation (see [8] for a more detailed discussion and a comparison between this technique and the use of higher-order modulations).

\footnotetext{
${ }^{1}$ Notice that our receiver processes the PM-QPSK signal components separately, thus it entails four two-state MAP detectors.
} 
There are other techniques based on tight filtering, induced intentional interference, and the adoption of advanced signal processing (e.g., see [12], [13]). For more references and a detailed comparison with TFP, the reader can refer to [8]. It is worth noting that even though many works report remarkable results on Super-Nyquist WDM systems using different techniques (e.g., see [14]-[20] for experimental results), our work for the first time presents results for a flexi-grid system heavily impaired by tight in-line filtering, and exploiting an information theoretic analysis to design the receiver processing. Recently, some works dealing with the computation of information rates (IRs) in optical communications were published, but the proposed methods are valid only for memoryless channels (e.g., see [21]), so they are not suitable for the analysis of a realistic scenario as the one presented in this work.

The paper is organized as follows. In Section II, a description of the system model link architecture and the adopted detection strategy are provided. Section III gives insight on the theoretical approach chosen to evaluate the receiver performance and to provide an analysis of system impairments. Section IV reports on the numerical results and the observations that arise. Finally, conclusions are drawn in Section V.

\section{SYSTEM MODEL}

The considered system is closely related to that described in [6]. In our WDM simulated system, $N_{c}$ PM linearly-modulated signals are launched on random polarizations and with random detuning with respect to central frequencies. In the following, we will consider a QPSK modulation format on each carrier and each polarization. The general expression for the complex envelope of the signal transmitted on the $\ell$ th carrier and the $i$ th polarization $(i=1,2)$ is

$$
\sum_{k=0}^{K-1} x_{k}^{(i, \ell)} p\left(t-k T-\tau^{(i, \ell)}\right) e^{j\left[2 \pi \ell\left(F+\Delta_{\ell}\right) t+\theta^{(i, \ell)}\right]}
$$

where $p(t)$ is the shaping pulse having root raised-cosine (RRC) spectrum with roll-off $\alpha=0.1$ (obtained by proper transmitside electrical filtering), $K$ the number of symbols transmitted over each carrier and each polarization, $T$ the symbol interval, $x_{k}^{(i, \ell)}$ the symbol transmitted over the $\ell$ th carrier of the $i$ th polarization during the $k$ th symbol interval, $\tau^{(i, \ell)}$ and $\theta^{(i, \ell)}$ the delay and the initial phase of the $i$ th polarization and $\ell$ th carrier, respectively, $F$ the frequency spacing between two adjacent carriers, and $\Delta_{\ell}$ the possible frequency offset (small compared to the frequency spacing). The transmitted symbols were obtained from a stream of information bits, by properly encoding with a binary FEC and Gray mapped onto the QPSK constellation.

The transmitted signal was then launched into a dispersion unmanaged fiber link with variable number of spans $N_{s}$, characterized by the presence of ROADM nodes, one every two fiber spans. Therefore, the number of crossed ROADMs was equal to half the number of spans. Each span had $120 \mathrm{~km}$ of single mode fiber (SMF) and an erbium-doped fiber amplifier (EDFA) with a noise figure of $6 \mathrm{~dB}$. Since ROADMs are here intended as simple pass-through nodes, they basically just in-

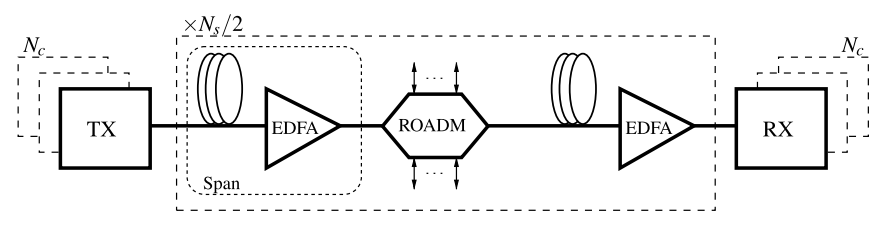

Fig. 1. Schematic of the simulated optical link.

troduce the filtering effect of two WSS, modeled as third-order super-Gaussian filters. The bandwidth (BW) of such filters can be determined once the flexible grid spacing has been selected. We fixed the channel spacing to $37.5 \mathrm{GHz}$, which implies a $3-\mathrm{dB}$ filter bandwidth of $35.75 \mathrm{GHz}$ [5], [6]. A span length of $120 \mathrm{~km}$ was chosen to worsen the system noise figure with respect to the more typical $100 \mathrm{~km}$ length and thus account for the WSS losses, and to avoid simulations of more than 100 spans, in order to keep the simulation time within reasonable limits.

Fiber propagation was impaired by group velocity dispersion (GVD), ${ }^{2}$ and nonlinear effects. These latter effects were simulated by the split step Fourier method (SSFM) [22] applied to the Manakov nonlinear equation with proper step size. ${ }^{3}$ The symbol rate $R$ of each signal was initially fixed to 32.5 Gbaud, as in [6]. Then, in order to demonstrate the advantages of the TFP technique [8], we increased the data rate beyond the Nyquist limit, up to 75 Gbaud, while keeping all remaining parameters unchanged (i.e., we did not change the filter bandwidths). Fig. 1 shows the block diagram of the generic simulated link.

At the receive side, coherent detection was performed [23]. The received optical field was first filtered by a fourth-order super-Gaussian filter having a 3-dB bandwidth of $35.75 \mathrm{GHz}$, which allows to select the desired channel, and then converted to the electrical domain through a $90^{\circ}$ optical hybrid. Digital signal processing was then performed, as explained in detail in [8]. After sampling, compensation of the cumulated GVD was performed by two fixed-tap equalizers (one per polarization) and then frame and frequency synchronization and compensation were performed. A two-dimensional fractionally-spaced adaptive minimum mean square error (MMSE) feed-forward equalizer (FFE) performed compensation of the residual GVD and polarization mode dispersion (PMD), and also performed polarization demultiplexing. The number of taps was chosen sufficiently high so that GVD and PMD did not entail any penalty, whereas a coefficient adaptation step-size value of $10^{-3}$ came up to be optimal in any case. Finally phase noise was tracked by a proper decision-directed phase estimation and compensation module. All synchronization aspects were neglected hereperfect synchronization was assumed. These aspects will be discussed in detail in a future paper. Finally, signal samples fed the detector, which, in the MAP case, iteratively exchanges soft information with the LDPC decoder.

\footnotetext{
${ }^{2}$ We also considered PMD with values of the differential group delay of typical fibers and noted no performance difference. Thus, PMD is not present in current results.

${ }^{3}$ We optimized the step-size for each launch power, by increasing the value in trial simulations until we noted no performance variation.
} 
We considered two kinds of detectors. First, we used a conventional symbol-by-symbol detector/demapper that neglects channel memory (i.e., the detector commonly used in coherent receivers). As a second more sophisticated solution, we employed a MAP symbol detector with a minimum number of states (see Section IV), preceeded by a channel shortener [24], which is essentially a linear filter with a few taps, and whose computation is based on the estimation of the overall channel impulse response. The shortener helps coping with the intersymbol interference (ISI) not accounted for by the limited detector memory, as explained in [8]. More details on the simulated system and receiver parameters will be given in Section IV.

On the described system, we performed two kinds of simulations. First, we evaluated the achievable SE, as explained in the next section, by varying the launch power per channel and number of spans $N_{s}$ of the link. Then, we also employed a set of codes with rates in the range 1/3-9/10. These codes were used to confirm the AIR semi-analytical predictions.

\section{SE ANALYSIS}

We now describe the framework used to evaluate the performance limits of all the optical transmission systems considered in this paper.

We consider an optical channel with linear and nonlinear distortions, simulated through the SSFM. Denoting by $\mathbf{y}$ a proper discrete-time sufficient statistic used for detection of the information symbols $\mathbf{x}=\left\{x_{k}^{(i, \ell)}\right\}_{k, i, \ell}$, with size $2 N_{c} K,{ }^{4}$ the IR, i.e., the average mutual information per symbol, is defined as

$$
\mathcal{I}=\lim _{K \rightarrow \infty} \frac{1}{2 N_{c} K} E\left\{\log _{2} \frac{p(\mathbf{y} \mid \mathbf{x})}{\sum_{\mathbf{x}^{\prime}} p\left(\mathbf{y} \mid \mathbf{x}^{\prime}\right) P\left(\mathbf{x}^{\prime}\right)}\right\}\left[\frac{\text { bit }}{\text { symbol }}\right]
$$

where $E[\cdot]$ denotes expectation, $p(\cdot)$ a probability density function (PDF) and $P(\cdot)$ the probability mass function (PMF) of $\mathrm{x}$. In this paper we always evaluate the IR when the information symbols are independent and uniformly distributed random variables belonging to the given constellation, so that $P(\mathbf{x})$ is a constant. The SE is the IR per unit bandwidth and unit time

$$
\mathrm{SE}=\frac{\mathcal{I}}{F T} \quad[\mathrm{~b} / \mathrm{s} / \mathrm{Hz}]
$$

since $F T$ is the time-frequency slot devoted to the transmission of symbol $x_{k}^{(i, \ell)}$.

The computation of IR and SE requires the availability of the PDFs $p(\mathbf{y} \mid \mathbf{x})$ and $p(\mathbf{y})=\sum_{\mathbf{x}^{\prime}} p\left(\mathbf{y} \mid \mathbf{x}^{\prime}\right) P\left(\mathbf{x}^{\prime}\right)$. However, they are not known in closed form, nor can we resort to the simulation method in [25] to compute them. In fact, this method requires that the channel at hand has a finite memory and the availability of an optimal detector for it [25]. These conditions are clearly not satisfied in our scenario [26], [27]. We may thus resort to the computation of a proper lower bound of the IR (and thus of the SE) obtained by substituting $p(\mathbf{y} \mid \mathbf{x})$ in (2) with an arbitrary auxiliary channel law $q(\mathbf{y} \mid \mathbf{x})$ with the same input and output alphabets as the original channel (mismatched detection [10],

\footnotetext{
${ }^{4}$ The factor 2 takes into account the presence of two polarizations.
}

[25], [28], [29]). The resulting lower bound reads as

$$
\mathcal{I}_{\mathrm{LB}}=\lim _{K \rightarrow \infty} \frac{1}{2 N_{c} K} E\left\{\log _{2} \frac{q(\mathbf{y} \mid \mathbf{x})}{\sum_{\mathbf{x}^{\prime}} q\left(\mathbf{y} \mid \mathbf{x}^{\prime}\right) P\left(\mathbf{x}^{\prime}\right)}\right\} .
$$

If the auxiliary channel law is representative of a finite-state channel, PDFs $q(\mathbf{y} \mid \mathbf{x})$ and $q_{p}(\mathbf{y})=\Sigma_{\mathbf{x}^{\prime}} q\left(\mathbf{y} \mid \mathbf{x}^{\prime}\right) P\left(\mathbf{x}^{\prime}\right)$ can be estimated by simulation, this time, by using the optimal MAP symbol detector for that auxiliary channel [25]. Such a detector, which will clearly be suboptimal for the actual channel, will have at its input the sequence $\mathbf{y}$ generated by simulation according to the actual channel model, and the expectation in (3) is taken with respect to the input and output sequences generated accordingly [25]. Thus, no assumption on the true statistics of the discrete-time received sequence is required for the design of the adopted detector, since it is designed for the auxiliary channel. Similarly, the true statistics of the sequence $\mathrm{y}$ are not analytically required for its generation, since they can be obtained by SSFM simulation through the actual nonlinear channel. If we change the auxiliary channel (and thus the trellis metrics based on it) we obtain different lower bounds on the IR but, in any case, such bounds are achievable by those receivers, according to mismatched detection [25], [28]. We will thus say that the computed lower bounds are the achievable SE values of the considered channel when those receivers are employed. All these considerations hold for any channel, including nonlinear and non-Gaussian ones.

This technique thus allows to evaluate the AIR for receivers of reduced complexity. In fact, it is sufficient to consider an auxiliary channel which is a simplified version of the actual channel in the sense that only a portion of the true channel memory and/or a limited number of impairments are present. The considered receivers have been described in the previous section. As mentioned, we have assumed that parallel independent detectors are employed, one for each carrier and each polarization. In other words, intercarrier interference (ICI) is not coped with at the receiver, since multiuser detection is considered too computationally demanding. This corresponds to the adoption of an auxiliary channel model that can be factorized into the product

$$
q(\mathbf{y} \mid \mathbf{x})=\prod_{i} \prod_{\ell} q\left(\mathbf{y}^{(i, \ell)} \mid \mathbf{x}^{(i, \ell)}\right)
$$

where $\mathbf{y}^{(i, \ell)}$ is a proper discrete-time received sequence used for detection of symbols $\mathbf{x}^{(i, \ell)}=\left\{x_{k}^{(i, \ell)}\right\}$ transmitted over the $\ell$ th carrier and the $i$ th polarization. Under this assumption, we simply have

$$
\mathcal{I}_{\mathrm{LB}}=\lim _{K \rightarrow \infty} \frac{1}{K} E\left\{\log _{2} \frac{q\left(\mathbf{y}^{(i, \ell)} \mid \mathbf{x}^{(i, \ell)}\right)}{q_{p}\left(\mathbf{y}^{(i, \ell)}\right)}\right\}
$$

i.e., the result can be computed by considering only one carrier. In a practical scenario with a finite number of carriers, we will consider the central carrier only, thus avoiding the computation on the border carriers which are affected by a smaller amount of ICI, thus obtaining a further lower bound.

Note that, as stated, we are not able to compute the IR of the true channel since the optimal receiver is unknown and possibly of unmanageable complexity. We take here the pragmatic 
approach of considering only limited-complexity suboptimal receivers. For such receivers we are indeed able to compute the relevant IR which will be called achievable IR. The corresponding achievable lower bound on SE (achievable SE in the following) is thus

$$
\eta_{\mathrm{LB}}=\frac{1}{F T} \mathcal{I}_{\mathrm{LB}} \quad[\mathrm{b} / \mathrm{s} / \mathrm{Hz}] .
$$

The auxiliary channel that we adopted for the MAP symbol detector design neglects the presence of channel nonlinear effects, and assumes that GVD and PMD have been perfectly compensated. Basically, the detector is designed by taking into account transmit side shaping pulse and in-line filtering, so that the sufficient statistics $\mathbf{y}^{(i, \ell)}$ can be obtained by sampling the output of a filter matched to the received pulse in the absence of GVD, PMD, and nonlinear effects, i.e., the transmit pulse after cascaded in-line filtering. ${ }^{5}$ In SSFM simulations, noise contributions introduced by EDFAs are added along the whole optical link at each span. Hence in-line filtering has an incremental effect on the propagating signal, whereas for the auxiliary channel we assume that all noise is added at the end of the link. Thus, at the receive side it is possible to estimate the overall channel response (e.g., through a simple MMSE estimator) without any knowledge on the link configuration, which corresponds to the most practical way to design the MAP symbol detector. Notice that the presence of other conventional receive-side filters, with bandwidth compatible with the chosen frequency grid, does not imply changes to the established matched filter response, thus does not affect the aforementioned considerations. Given this auxiliary channel law, the optimal MAP symbol detector is described in [30] (see [8] for more details).

As a concluding remark, we would like to point out that this technique allows to compute the achievable limit of the considered receivers without taking into account specific coding schemes, being understood that, with a properly designed channel code, the information-theoretic performance can be closely approached. Section IV will report some design cases for these codes with the aim of showing that, indeed, the performance predicted by the achievable SE can be approached.

\section{NUMERICAL RESULTS}

In this section, we present the simulation results of the optical channel described in Section II. The WDM input signal in (1) had 11 channels with spacing $37.5 \mathrm{GHz}$ and was launched in the line with $P$ power per channel and propagated through a dispersion-uncompensated (DU) link of $N_{s}$ identical $120 \mathrm{~km}$ SMF spans. Fiber parameters include dispersion $16.63 \mathrm{ps} / \mathrm{nm} / \mathrm{km}$, attenuation $0.23 \mathrm{~dB} / \mathrm{km}$, and nonlinear index $\gamma=1.3 \mathrm{~W}^{-1} \cdot \mathrm{km}^{-1}$. Every two spans we included a ROADM, whose only effect is a filtering due to the presence of two WSS on the signal path. In Table I, we recall system filter types and bandwidths, which are kept unchanged in all

\footnotetext{
${ }^{5}$ The FFE taps are designed by using the matched filter output as the target response, so that the equalizer does not remove the ISI induced by filtering but only performs matched filtering. It is worth noting that, if extremely narrow optical filtering is employed at the receive side, the electrical compensation of chromatic dispersion through the non-adaptive equalizers may be inaccurate. In this case, a wider optical filter can be used, compatibly with the system design, in
}

TABLE I

FILTERS PARAMETERS

\begin{tabular}{lcccc}
\hline \hline Type & $\begin{array}{c}\text { TX Optical } \\
\text { RRC, } \alpha=0.1\end{array}$ & $\begin{array}{c}\text { In-line WSS } \\
\text { Third Gauss. }\end{array}$ & $\begin{array}{c}\text { RX Optical } \\
\text { Fourth Gauss. }\end{array}$ & $\begin{array}{c}\text { RX electrical } \\
\text { Fifth Bessel }\end{array}$ \\
\hline 3-dB BW [GHz] & 35.75 & 35.75 & 35.75 & 16 \\
\hline \hline
\end{tabular}

TABLE II

CONCATENATED BANDWIDTH VERSUS CROSSED WSS

\begin{tabular}{lccccccc}
\hline \hline \# WSS & 2 & 4 & 8 & 12 & 16 & 32 & 64 \\
3-dB CBW [GHz] & 31.85 & 28.4 & 25.2 & 23.6 & 22.5 & 20 & 17.8 \\
\hline
\end{tabular}

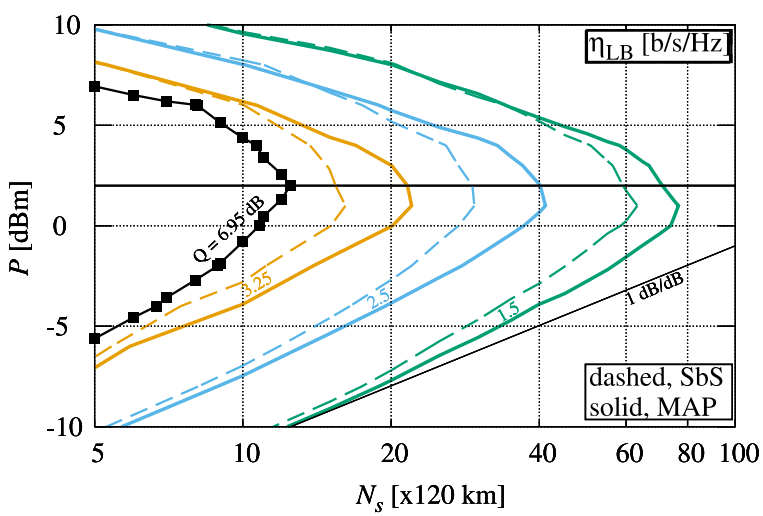

Fig. 2. Horizontal cuts of SE versus launch power per channel and number of spans $N_{s}$, in a $N_{s} \times 120 \mathrm{~km} \mathrm{SMF} \mathrm{DU} \mathrm{link,} \mathrm{with} R=32.5$ Gbaud, $F=$ $37.5 \mathrm{GHz}$, MAP detector with $L=1$ and conventional symbol-by-symbol detector. It is also reported the horizontal cut corresponding to $\mathrm{BER}=0.0132$, or Q-factor $=6.95 \mathrm{~dB}$, assumed as a pre-FEC threshold for a $25 \%$ overhead code, symbol-by-symbol detector.

simulations. The effect of cascading WSS is then summarized in Table II, which reports the concatenated bandwidth (CBW), versus the number of crossed WSS.

We simulated the propagation of $R=32.5$ Gbaud channels, and each of them was detected by using proper MAP symbol detectors which take into account a memory of $L=1$ symbols. Since we use a QPSK modulation per polarization, the detector was split into four binary detectors with $2^{L}=2$ states, each operating on one polarization and one quadrature. In addition, we also considered the use of a standard symbol-by-symbol detector. Results are shown in Fig. 2, where horizontal cuts of SE surfaces are plotted versus $P$ and $N_{s}{ }^{6}{ }^{6}$ For each point of the surface we averaged over six clusters of about 70000 symbols, and obtained a confidence interval of at worst $4 \%$. Furthermore, for each point the transmitted channels were launched with random initial polarization, time delays, and offset frequencies. As far as the frequency offset is concerned, it can be considered to be two orders of magnitude smaller than the carrier spacing. In our simulations we included random offsets in the range $\pm 1 \%$

order to leave the useful component of the received signal unchanged, whereas matched filtering is implemented by the adaptive equalizer.

${ }^{6}$ Notice that, as we are computing the IR, it is unnecessary to also present BER curves for the considered systems, since information theory states that an arbitrarily small BER can be reached using a capacity-achieving code with rate lower than a given IR value. 


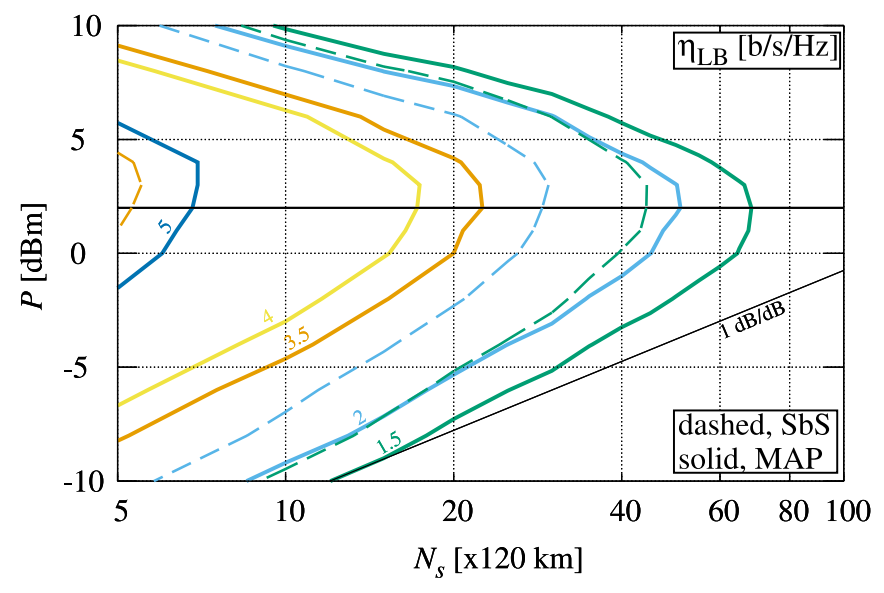

Fig. 3. Horizontal cuts of SE versus launch power per channel and number of spans $N_{s}$, in a $N_{s} \times 120 \mathrm{~km}$ SMF DU link, with $R=50$ Gbaud, $F=$ $37.5 \mathrm{GHz}$, MAP detector with $L=1$ and conventional symbol-by-symbol detector.

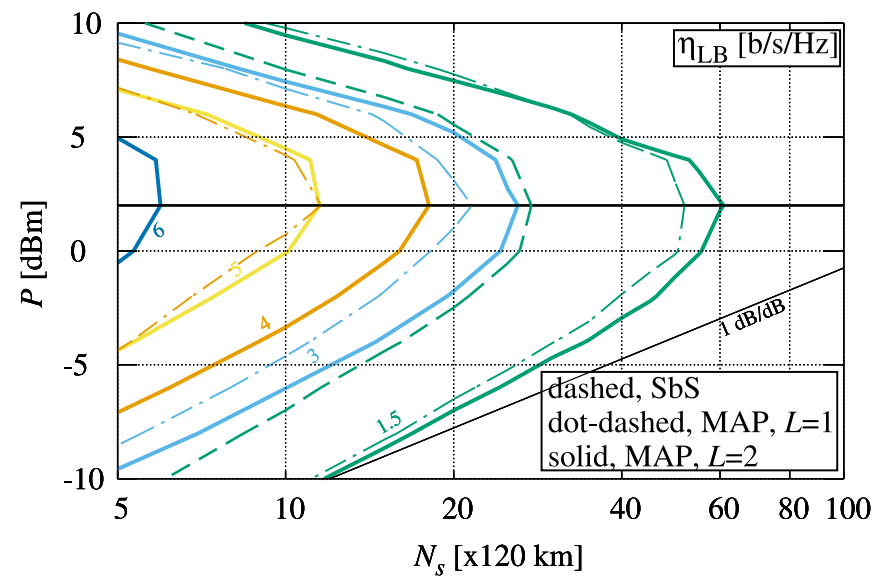

Fig. 4. Horizontal cuts of SE versus launch power per channel and number of spans $N_{s}$, in a $N_{s} \times 120 \mathrm{~km} \mathrm{SMF} \mathrm{DU} \mathrm{link,} \mathrm{with} R=75$ Gbaud, $F=$ 37.5 GHz, MAP detector with $L=1$ and $L=2$ and conventional symbol-bysymbol detector.

of the carrier spacing. This choice is not dictated by the need to perform carrier synchronization. In fact, plenty of algorithms able to recover a frequency offset up to $30-40 \%$ of the symbol rate are available in the literature (e.g., see [31] and references therein). On the other hand, with the tight filters used in flexi-grid systems, a larger frequency offset would have a negative impact on the signal power at the filters' output. So a more strict control on the frequency instabilities must be adopted with respect to classical systems. Nevertheless, we also performed a few simulations with frequency offsets up to $\pm 5 \%$ of the carrier spacing, observing negligible penalty. We also report in this figure the horizontal cut corresponding to an uncoded BER of 0.0132 (or, equivalently, a Q-factor equal to $6.95 \mathrm{~dB}$ ) for the symbol-bysymbol detector, which represents a conventional pre-FEC BER for a $25 \%$ overhead code (to be more precise, a concatenated $\mathrm{BCH}$ code, see [32]). From the figures, it can be noticed that the theoretical SE $\eta_{\mathrm{LB}}$ (back-to-back case) of $\sim 4 R / F[\mathrm{~b} / \mathrm{s} / \mathrm{Hz}]$ decreases with increasing distance, and MAP detector shows a clear maximum reach advantage. The gain of the MAP detec-

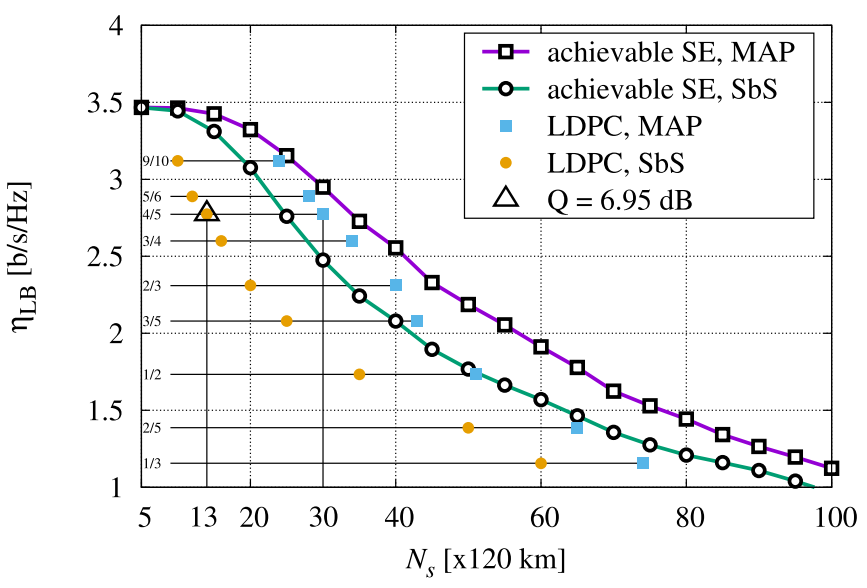

Fig. 5. SE versus number of spans, at $2 \mathrm{dBm}$ launch power, $R=32.5 \mathrm{Gbaud}$ $F=37.5 \mathrm{GHz}$, symbol-by-symbol ( $\mathrm{SbS}$ ) and MAP detectors, and simulations with rate 1/3, 2/5, 1/2, 3/5, 2/3,3/4, 4/5, 5/6, 9/10 LDPC codes (with reference BER $=10^{-4}$ ). The triangle refers to the pre-FEC Q-factor for a $25 \%$ overhead code.

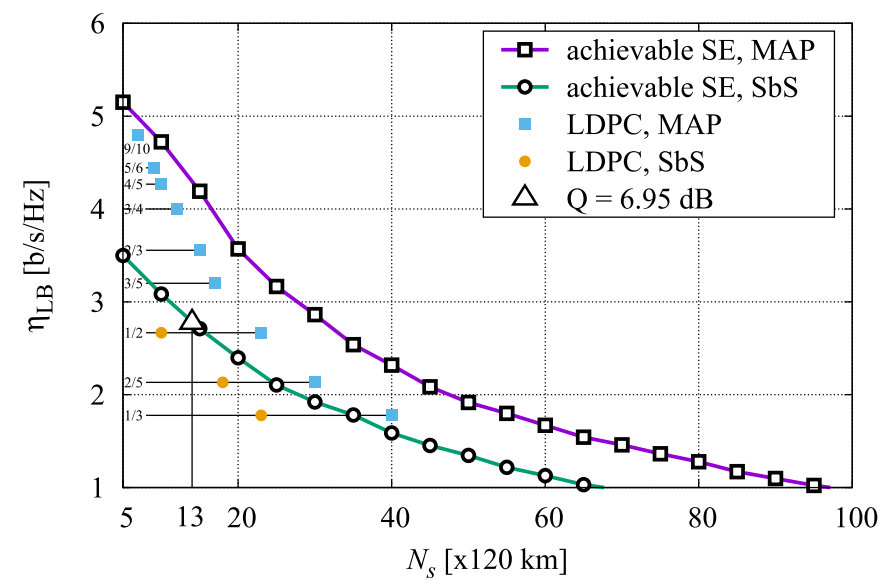

Fig. 6. SE versus number of spans, at $2 \mathrm{dBm}$ launch power, $R=50 \mathrm{Gbaud}$, $F=37.5 \mathrm{GHz}$, symbol-by-symbol (SbS), MAP detectors and simulations with rate $1 / 3,2 / 5,1 / 2,3 / 5,2 / 3,3 / 4,4 / 5,5 / 6,9 / 10$ LDPC codes (with reference BER $\left.=10^{-4}\right)$. The triangle refers to the pre-FEC Q-factor for a $25 \%$ overhead code.

tor can be explained with the improved performance in linear regime, where the limited memory of the channel is properly exploited by the detector. Such a gain decreases in nonlinear regime since the ISI introduced by in-line filtering is masked by the huge memory (not accounted for in the auxiliary channel) brought by the nonlinear channel. Nevertheless, the gain of the MAP detector is still significant because at the optimal launch power optical noise is twice as important as nonlinear interference noise [33]. It is worth noting that the slope of these contour plots in nonlinear regime are in good agreement with curves shown in [33] (but it is not equal to $1 \mathrm{~dB} / \mathrm{dB}$ in linear regime due to in-line filtering), where the Gaussian-noise model for DU optical systems [34] was assumed, and signal-to-noise ratio (SNR) cuts are drawn versus $P$ and $N_{s}$.

Fig. 3 presents the same horizontal cuts of SE surfaces, for the case $R=50$ Gbaud. We are here in the realm of TFP [8], [35]. The shape of the contour plots are very similar to those in the previous figure but, in this case, it is possible to appreciate 


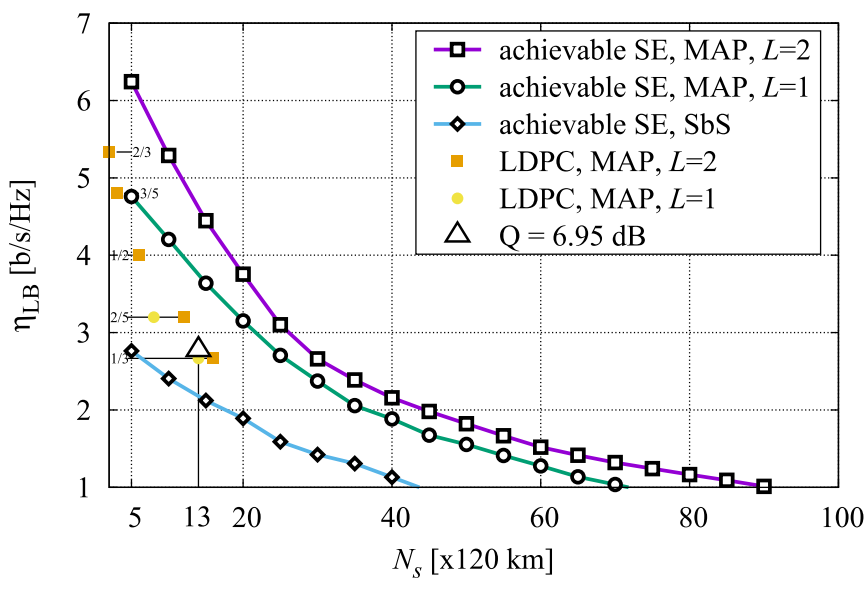

Fig. 7. SE versus number of spans, at $2 \mathrm{dBm}$ launch power, $R=75$ Gbaud, $F=37.5 \mathrm{GHz}$, symbol-by-symbol ( $\mathrm{SbS})$, MAP detectors and simulations with rate $1 / 3,2 / 5,1 / 2,3 / 5,2 / 3$ LDPC codes (with reference BER $=10^{-4}$ ). The triangle refers to the pre-FEC Q-factor for a $25 \%$ overhead code.

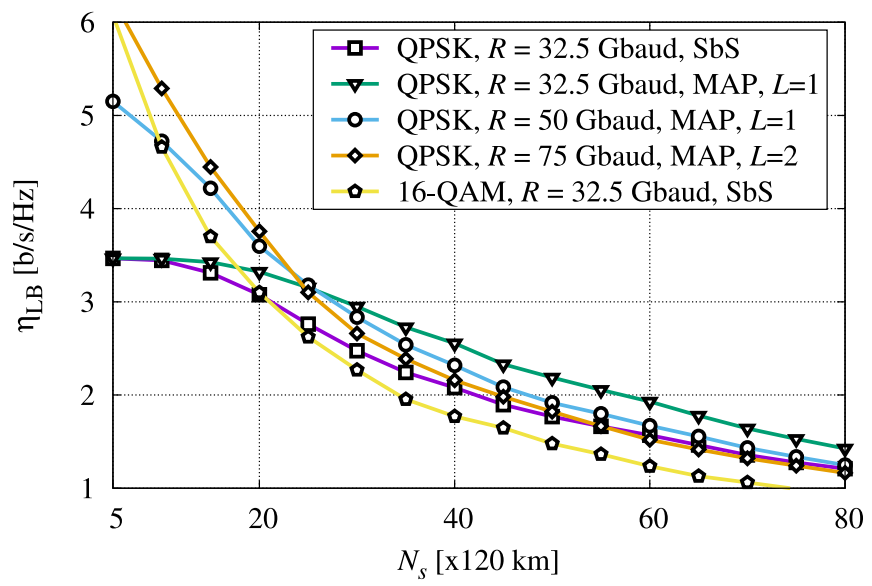

Fig. 8. SE versus number of spans $N_{s}$, at $2 \mathrm{dBm}$ launch power, for $R=$ 32.5 Gbaud, $R=50$ Gbaud and $R=75$ Gbaud, $F=37.5 \mathrm{GHz}$, symbol-bysymbol (SbS) and MAP detectors, QPSK and 16-QAM modulations.

the relevant SE improvement (a back-to-back theoretical value of $\eta_{\mathrm{LB}} \simeq 5.35[\mathrm{~b} / \mathrm{s} / \mathrm{Hz}]$ can be observed at the optimal power), which outlines a clear benefit with MAP symbol detector especially for short distances. On the contrary, the threshold detector performs worse than in the $R=32.5$ Gbaud case already after a few spans, as expected (since it is not able to cope with the intentional ISI introduced by transmit-side narrow filtering). Fig. 4 further shows results at $R=75$ Gbaud, that is twice the channel spacing. In this case, since the packing is denser, we also plotted curves for $L=2$ (i.e., four states). A significant SE improvement can be noticed at shorter distances, where $\eta_{\mathrm{LB}}$ higher than $6 \mathrm{~b} / \mathrm{s} / \mathrm{Hz}$ is reached. In this scenario, clearly, the symbol-by-symbol dector performs poorly, reaching at most $\eta_{\mathrm{LB}} \simeq 1.5 \mathrm{~b} / \mathrm{s} / \mathrm{Hz}$. Notice that in Figs. 3 and 4 the cut corresponding to the Q-factor of $6.95 \mathrm{~dB}$ is not present, because it falls below 5 spans.

Figs. 5-7 show instead the vertical cuts of the SE surfaces at $P=2 \mathrm{dBm}$, for $R=32.5$ Gbaud, $R=50$ Gbaud and $R=$
75 Gbaud, respectively. For this scenario, we also simulated coded signaling, by using LDPC codes with rates from $1 / 3$ to 9/10 from [9], and declaring the maximum reach at distances where an estimated post-FEC BER lower than $10^{-4}$ was achieved, which in practice implies convergence of the iterative detection/decoding algorithm. ${ }^{7}$ We fixed a limit of 40 iterations, and averaged over 500000 received symbols per step. We found a good agreement between expected results from achievable lower bounds and simulations, with more affinity for the MAP detector at small distances, since in this case the auxiliary channel assumed by the receiver is closer to the true channel (i.e., the effect of cascaded ROADMs is still not critical). However, the gap between achievable SE and simulations becomes larger when the system is more impaired by ISI and at the same time the actual and auxiliary channels are more divergent, as can be inferred from Fig. 7. In this case, the system would benefit from a careful re-design of the employed codes. In any case, simulations with LDPC codes confirm the reliability of the SE analysis performed through the AIR lower bounds computation. It is interesting to notice that our simulated rate- $4 / 5$ code with the $\mathrm{SbS}$ detector has the same performance as the 6.95-dB reference code indicated by the triangle in Fig. 5, whereas if the same code is used with the MAP detector the reach can be more than doubled. From Figs. 5-7, it can be observed that the larger the number of spans, the larger the difference between the theoretical performance and the results achieved by the considered practical codes. This is related to the fact that these codes have been designed and optimized for a channel without interference. When the number of spans becomes too large, a code redesign becomes necessary to adapt the degree distributions of the LDPC codes to these channels with strong interference. This can be performed by using standard techniques based on the Extrinsic Information Transfer charts [36]. Once the degree distributions have been optimized, the algorithm in [37] can then be used for the generation of the code parity check matrix.

Finally, in Fig. 8 we report the curves of Figs. 5-7, in order to provide a direct and clearer comparison of the results. We also included simulations of the 16-quadrature amplitude modulation (QAM) format with the symbol-by-symbol detector at $R=$ 32.5 Gbaud, just to place our results in a perspective with respect to a known commercial reference system, without making direct comparisons which would be unfair in this case. At equal symbol rate, being the launched signal only slightly affected by ISI, benefits are remarkable when the effect of in-line WSS filtering starts to significantly impair the signal. On the other hand, if we consider the 50 and 75 Gbaud signals, an impressive SE gain up to $80 \%$ is obtained at smaller distances, while there is no gain after about 25 spans, i.e. $3000 \mathrm{~km}$. In order to further highlight the effect of tight transmission filtering on the signal, Fig. 9 shows the signal constellation on one polarization for a

\footnotetext{
${ }^{7}$ This reference BER of $10^{-4}$ has been clearly selected to reduce the simulation time. However, since these codes have a very steep waterfall, a BER below $10^{-7}$ can be achieved at a SNR value of just a fraction of $\mathrm{dB}$ higher. A much lower BER cannot be achieved with these codes since they have a an error floor around $10^{-7}-10^{-8}$. From this BER, additional outer codes with $2-3 \%$ overhead, tipically $\mathrm{BCH}$, can further reduce the BER down to $10^{-12}$ [9].
} 


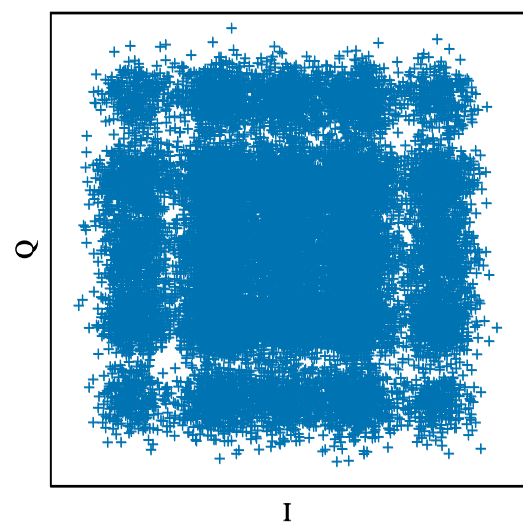

Fig. 9. Signal constellation of one polarization for a 50 Gbaud QPSK signal after 5 spans at $2 \mathrm{dBm}$ launch power.

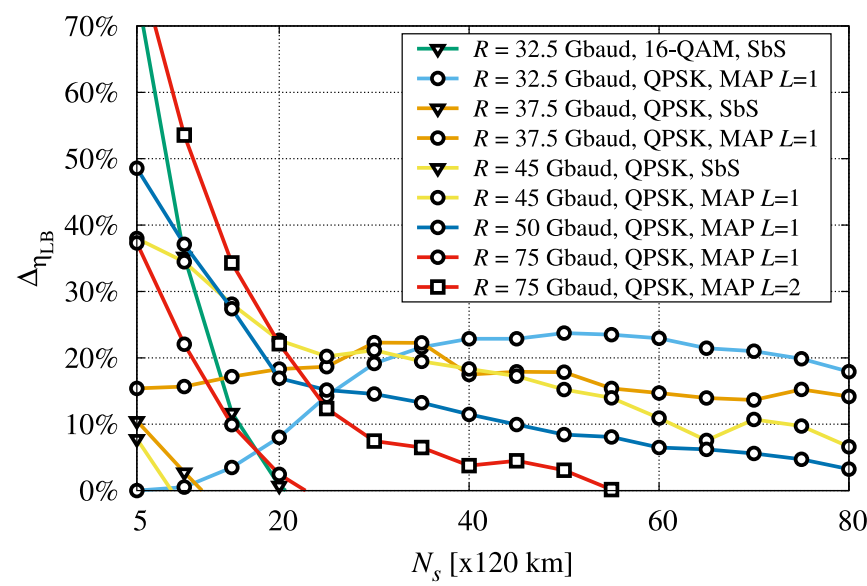

Fig. 10. Relative SE increment with respect to conventional symbol-bysymbol detector at $R=32.5$ Gbaud, versus number of spans $N_{s}$. The MAP curves refer to all simulated symbol rates, from $R=32.5$ Gbaud to $R=75$ Gbaud.

50 Gbaud signal after 5 spans, considering a launch power of $2 \mathrm{dBm}$.

By defining the relative $\mathrm{SE}$ gain of the MAP receiver with respect to the $\mathrm{SbS}$ receiver as

$$
\Delta_{\eta_{\mathrm{LB}}}=\frac{\eta_{\mathrm{LB}}-\eta_{\mathrm{LB}, \mathrm{REF}}}{\eta_{\mathrm{LB}, \mathrm{REF}}}
$$

where $\eta_{\mathrm{LB}, \mathrm{REF}}$ is the achievable SE value of the QPSK symbolby-symbol detector with $R=32.5$ Gbaud at each distance, in Fig. 10 we plot the relative gain in SE of MAP detector versus distance $N_{s}$ in variable symbol rate scenarios (we simulated $32.5,37.5,45,50,75$ Gbaud channels, in the latest case with $L=2$ ), highlighting the benefits of the chosen 2- and 4-state detectors. From the individual curves, an envelope of all simulated symbol rates can be determined, so it is possible to infer that choosing a suitable symbol rate depending on the link length allows to keep a consistent SE gain with respect to a conventional symbol-by-symbol detector. Then, by examining the single curves, it can be noticed that at longer distances the best choice turns out to be the lowest rate, as can be expected by its natural tolerance to nonlinear effects and tight filtering. In the mid distance range, around $N_{s}=20$, on the other hand, the MAP QPSK shows a consistent gain with respect also to the symbol-by-symbol 16-QAM, whereas in the short range (i.e., however, up to $2400 \mathrm{~km}$ ) a remarkable gain can be observed for $R=75$ Gbaud and MAP with $L=2$, confirming that QPSK with a four-state MAP detector can even outperform high-order modulations (see[8] for comparisons at a fixed complexity).

\section{CONCLUSION}

In this paper we investigated the effects of narrow filtering in WDM transmission over flexible grid optical networks, with particular emphasis on $37.5 \mathrm{GHz}$ spacing and 32.5 symbol rate with the QPSK modulation format. We showed that it is possible to mitigate the detrimental ISI introduced by cascading WSS along the link by employing a two-state MAP symbol detector for each input bit stream. The mitigation is quantified in terms of achievable SE versus propagation distance and launch power. Moreover, we proved that it is possible to exploit LDPC codes of different rates in order to achieve a desired SE, thus obtaining a remarkable system reach improvement, or, conversely, higher $\mathrm{SE}$ at equal reach, with respect to the conventional symbolby-symbol detector. Finally, we proved that by transmitting 50 Gbaud channels on the same DU SMF link a great SE gain can be achieved by working beyond the Nyquist-WDM limit through TFP, which enables a remarkable SE gain for distances up to $3000 \mathrm{~km}$.

\section{REFERENCES}

[1] G. Bosco, V. Curri, A. Carena, P. Poggiolini, and F. Forghieri, "On the performance of Nyquist-WDM terabit superchannels based on PM-QPSK, PM-8PSK or PM-16QAM subcarriers," J. Lightw. Technol., vol. 29, no. 1, pp. 53-61, Jan. 2011.

[2] S. L. Jansen, B. Spinnler, I. Morita, S. Randel, and H. Tanaka, "100GbE: QPSK versus OFDM," Opt. Fiber Technol., vol. 15, no. 5-6, pp. 407-413, Oct-Dec. 2009.

[3] R. Tkach and A. Chraplyvy, "Past/present system advances, with an eye towards the future," in Proc. Eur. Conf. Opt. Commun., Sep. 2010, pp. 1-10.

[4] Spectral Grids for WDM Applications: DWDM Frequency Grid, ITU-T G.694.1, Feb. 2012.

[5] Finisar, "Balancing performance, flexibility, and scalability in optical networks," White Paper, Feb. 2012.

[6] A. Morea, J. Renaudier, A. Ghazisaeidi, O. Bertrand-Pardo, and T. Zami, "Impact of reducing channel spacing from $50 \mathrm{GHz}$ to $37.5 \mathrm{GHz}$ in fully transparent meshed networks," presented at the Opt. Fiber Commun. Conf., San Francisco, CA, USA, Mar. 2014, Paper Th1E.4.

[7] L. R. Bahl, J. Cocke, F. Jelinek, and J. Raviv, "Optimal decoding of linear codes for minimizing symbol error rate," IEEE Trans. Inf. Theory, vol. IT-20, no. 2, pp. 284-287, Mar. 1974.

[8] G. Colavolpe and T. Foggi, "Time-frequency packing for high capacity coherent optical links," IEEE Trans. Commun., vol. 62, no. 8, pp. 29862995, Aug. 2014.

[9] ETSI EN 301307 Digital Video Broadcasting (DVB); V1.1.2 (2006), Second generation framing structure, channel coding and modulation systems for Broadcasting, Interactive Services, News Gathering and other Broadband satellite applications. [Online]. Available: http://www.etsi.org

[10] G. Colavolpe, T. Foggi, A. Modenini, and A. Piemontese, "Faster-thanNyquist and beyond: how to improve spectral efficiency by accepting interference," Opt. Exp., vol. 19, no. 27, pp. 26600-26609, Dec. 2011.

[11] A. Barbieri, D. Fertonani, and G. Colavolpe, "Spectrally-efficient continuous phase modulations," IEEE Trans. Wireless Commun., vol. 8, no. 3, pp. 1564-1572, Mar. 2009.

[12] J. Li, Z. Tao, H. Zhang, W. Yan, T. Hoshida, and J. C. Rasmussen, "Spectrally efficient quadrature duobinary coherent systems with symbolrate digital signal processing," J. Lightw. Technol., vol. 29, no. 8, pp. 1098-1104, Apr. 2011. 
[13] J. Li, E. Tipsuwannakul, T. Eriksson, M. Karlsson, and P. A. Andrekson, "Approaching Nyquist limit in WDM systems by low-complexity receiver-side duobinary shaping," J. Lightw. Technol., vol. 30, no. 11, pp. 1664-1676, Jun. 2012.

[14] K. Kikuchi, Y. Ishikawa, and K. Katoh, "Coherent demodulation of optical quadrature duobinary signal with spectral efficiency of $4 \mathrm{bit} / 51 \mathrm{hz}$ per polarization," presented at the Eur. Conf. Optical Commun., Berlin, Germany, Sep. 2007, Paper 9.3.4.

[15] I. Lyubomirsky, "Quadrature duobinary for high-spectral efficiency $100 \mathrm{~g}$ transmission," J. Lightw. Technol., vol. 28, no. 1, pp. 91-96, Jan. 2010.

[16] J. Zhang and X. Li, "Improved quadrature duobinary system performance using multi-modulus equalization," IEEE Photon. Technol. Lett., vol. 25, no. 16, pp. 1630-1633, Aug. 2013.

[17] J.-X. Cai, Y. Cai, C. R. Davidson, F. D. G., L. A. J., O. V. Sinkin, W. W. Patterson, A. N. Pilipetskii, G. Mohs, and N. S. Bergano, "Transmission of 96 100-gb/s bandwidth-constrained PDM-RZ-QPSK channels with 300 spectral efficiency over $10610 \mathrm{~km}$ and $400 \mathrm{~km}$," J. Lightw. Technol., vol. 29, no. 4, pp. 491-498, Feb. 2011.

[18] X. Cai, C. R. Davidson, A. Lucero, H. Zhang, D. G. Foursa, O. V. Sinkin, W. W. Patterson, A. N. Pilipetskii, G. Mohs, and N. S. Bergano, "20 tbit/s transmission over $6860 \mathrm{~km}$ with sub-Nyquist channel spacing," J. Lightw. Technol., vol. 30, no. 4, pp. 651-657, Feb. 2012.

[19] K. Igarashi, T. Tsuritani, I. Morita, Y. Tsuchida, K. Maeda, M. Tadakuma, T. Saito, K. Watanabe, K. Imamura, R. Sugizaki, and M. Suzuki, "1.03exabit/s*km super-nyquist-WDM transmission over 7,326-km seven-core fiber," presented at the Eur. Conf. Opt. Commun., London, U.K., Sep. 2013, Paper PD3.E.3.

[20] J. Renaudier, R. Rios-Muller, L. Schmalen, M. Salsi, P. Tran, G. Charlet, and S. Bigo, "1-Tb/s transceiver spanning over just three $50-\mathrm{GHz}$ frequency slots for long-haul systems," presented at the Eur. Conf. Optical Commun., London, U.K., Sep. 2013, Paper PD2.D.5.

[21] M. Secondini, E. Forestieri, and G. Prati, "Achievable information rate in nonlinear WDM fiber-optic systems with arbitrary modulation formats and dispersion maps," J. Lightw. Technol., vol. 31, no. 23, pp. 3839-3852, Dec. 2013

[22] O. V. Sinkin, R. Holzlöhner, J. Zweck, and C. R. Menyuk, "Optimization of the split-step fourier method in modeling optical-fiber communication systems," J. Lightw. Technol., vol. 21, no. 1, pp. 61-68, Jan. 2003.

23] G. Colavolpe, T. Foggi, E. Forestieri, and G. Prati, "Multilevel optica systems with MLSD receivers insensitive to GVD and PMD," J. Lightw. Technol., vol. 26, no. 10, pp. 1263-1273, May 2008.

[24] F. Rusek and A. Prlja, "Optimal channel shortening for MIMO and ISI channels," IEEE Trans. Wireless Commun., vol. 11, no. 2, pp. 810-818, Feb. 2012.
[25] D. M. Arnold, H.-A. Loeliger, P. O. Vontobel, A. Kavčić, and W. Zeng, "Simulation-based computation of information rates for channels with memory," IEEE Trans. Inf. Theory, vol. 52, no. 8, pp. 3498-3508, Aug. 2006

[26] R. Essiambre and R. W. Tkach, "Capacity trends and limits of optical communication networks," Proc. IEEE, vol. 100, no. 5, pp. 1035-1055, May 2012.

[27] R. Essiambre, G. Kramer, P. J. Winzer, G. J. Foschini, and B. Goebel, "Capacity limits of optical fiber networks," J. Lightw. Technol., vol. 28, no. 4, pp. 662-701, Feb. 2010

[28] N. Merhav, G. Kaplan, A. Lapidoth, and S. Shamai, "On information rates for mismatched decoders," IEEE Trans. Inf. Theory, vol. 40, no. 6, pp. 1953-1967, Nov. 1994.

[29] P. Serena, A. Bononi, and G. Colavolpe, "On the nonlinear capacity with memory of PS-QPSK and PDM-QPSK in WDM non-dispersion managed links," presented at the Eur. Conf. Opt. Commun., Amsterdam, The Netherlands, Sep. 2012, Paper We.2.C.1.

[30] G. Colavolpe and A. Barbieri, "On MAP symbol detection for ISI channels using the Ungerboeck observation model," IEEE Commun. Lett., vol. 9, no. 8, pp. 720-722, Aug. 2005

[31] U. Mengali and M. Morelli, "Data-aided frequency estimation for burst digital transmission," IEEE Trans. Commun., vol. 45, no. 1, pp. 23-25, Jan. 1997.

[32] Forward Error Correction for High Bit-Rate DWDM Submarine Systems, ITU-T G.675.1, Feb. 2004

[33] A. Bononi, N. Rossi, and P. Serena, "On the nonlinear threshold versus distance in long-haul highly-dispersive coherent systems," Opt. Exp., vol. 20, no. 26, pp. 204-216, Dec. 2012.

[34] A. Carena, V. Curri, G. Bosco, P. Poggiolini, and F. Forghieri, "Modeling of the impact of nonlinear propagation effects in uncompensated optical coherent transmission links," J. Lightw. Technol., vol. 30, no. 10, pp. 1524-1539, May 2012.

[35] A. Barbieri, D. Fertonani, and G. Colavolpe, "Time-frequency packing for linear modulations: Spectral efficiency and practical detection schemes," IEEE Trans. Commun., vol. 57, no. 10, pp. 2951-2959, Oct. 2009.

[36] S. ten Brink, G. Kramer, and A. Ashikhmin, "Design of low-density parity-check codes for modulation and detection," IEEE Trans. Commun., vol. 52, no. 4, pp. 670-678, Apr. 2004.

[37] H. Xiao and A. H. Banihashemi, "Improved progressive-edge-growth (PEG) construction of irregular LDPC codes," IEEE Commun. Lett., vol. 8, no. 12, pp. 715-717, Dec. 2004.

Authors' biographies not available at the time of publication. 